\title{
BMJ Open Diagnostic workup for fever of unknown origin: a multicenter collaborative retrospective study
}

Toshio Naito, ${ }^{1}$ Masafumi Mizooka, ${ }^{2}$ Fujiko Mitsumoto, ${ }^{3}$ Kenji Kanazawa, ${ }^{4}$ Keito Torikai, ${ }^{5}$ Shiro Ohno, ${ }^{6}$ Hiroyuki Morita, ${ }^{7}$ Akira Ukimura, ${ }^{8}$ Nobuhiko Mishima, ${ }^{9}$ Fumio Otsuka, ${ }^{10}$ Yoshio Ohyama, ${ }^{11}$ Noriko Nara, ${ }^{12}$ Kazunari Murakami, ${ }^{13}$ Kouichi Mashiba, ${ }^{14}$ Kenichiro Akazawa, ${ }^{15}$ Koji Yamamoto, ${ }^{16}$ Shoichi Senda, ${ }^{17}$ Masashi Yamanouchi, ${ }^{1}$ Susumu Tazuma, ${ }^{2}$ Jun Hayashi ${ }^{3}$

To cite: Naito T, Mizooka M, Mitsumoto $\mathrm{F}$, et al. Diagnostic workup for fever of unknown origin: a multicenter collaborative retrospective study. BMJ Open 2013;3:e003971. doi:10.1136/bmjopen-2013003971

- Prepublication history for this paper is available online. To view these files please visit the journal online (http://dx.doi.org/10.1136/ bmjopen-2013-003971).

CrossMark

For numbered affiliations see end of article.

Correspondence to

Dr Toshio Naito;

naito@juntendo.ac.jp

\section{ABSTRACT}

Objective: Fever of unknown origin (FUO) can be caused by many diseases, and varies depending on region and time period. Research on FUO in Japan has been limited to single medical institution or region, and no nationwide study has been conducted. We identified diseases that should be considered and useful diagnostic testing in patients with FUO.

Design: A nationwide retrospective study.

Setting: 17 hospitals affiliated with the Japanese Society of Hospital General Medicine.

Participants: This study included patients $\geq 18$ years diagnosed with 'classical fever of unknown origin' (axillary temperature $\geq 38^{\circ} \mathrm{C}$ at least twice over a $\geq 3$-week period without elucidation of a cause at three outpatient visits or during 3 days of hospitalisation) between January and December 2011.

Results: A total of 121 patients with FUO were enrolled. The median age was 59 years (range 19-94 years). Causative diseases were infectious disease in 28 patients $(23.1 \%)$, non-infectious inflammatory disease in $37(30.6 \%)$, malignancy in $13(10.7 \%)$, other in $15(12.4 \%)$ and unknown in $28(23.1 \%)$. The median interval from fever onset to evaluation at each hospital was 28 days. The longest time required for diagnosis involved a case of familial Mediterranean fever. Tests performed included blood cultures in $86.8 \%$, serum procalcitonin in $43.8 \%$ and positron emission tomography in $29.8 \%$ of patients.

Conclusions: With the widespread use of CT, FUO due to deep-seated abscess or solid tumour is decreasing markedly. Owing to the influence of the ageing population, polymyalgia rheumatica was the most frequent cause (9 patients). Four patients had FUO associated with HIV/AIDS, an important cause of FUO in Japan. In a relatively small number of cases, cause remained unclear. This may have been due to bias inherent in a retrospective study. This study identified diseases that should be considered in the differential diagnosis of FUO.

\section{Strengths and limitations of this study}

This study is the first nationwide study of patients with fever of unknown origin in Japan.

- The present study identified diseases that should be considered in the differential diagnosis of fever of unknown origin.

- The rate of 'unknown' in our study was relatively small, but this retrospective study may have encountered bias because of the difficulty of enrolling patients with an unknown cause after undergoing tests.

\section{INTRODUCTION}

Fever of unknown origin (FUO) can be caused by many diseases, and causes can vary depending on region and time period. FUO was first reported in the medical literature 80 years ago. ${ }^{1}$ Since then, the causative diseases have greatly changed with changes in the social environment and widespread use of diagnostic imaging. ${ }^{2-6}$

A few reports of FUO have also been published in Japan, but have been limited to single-facility or limited-region studies ${ }^{7}$; no nationwide studies have yet been conducted. Moreover, few assessments of tests used in the diagnostic evaluation of FUO have been reported. In particular, few studies have assessed the clinical usefulness of tests such as serum procalcitonin or positron emission tomography (PET) in Japan, although these tests are now frequently used.

We therefore conducted a multicenter collaborative retrospective study of patients with FUO at hospitals affiliated with the Japanese Society of Hospital General Medicine. This is the first nationwide study in Japan on diseases causing FUO and the diagnostic workup, and identified diseases that should be considered when evaluating FUO in 
Japan. In addition, we investigated the rate of performing various tests in the current diagnostic workup of FUO.

\section{METHODS}

Among 99 hospitals affiliated with the Japanese Society of Hospital General Medicine that were asked to participate in this study, 17 hospitals participated. The hospitals participated in our study had a wide geographic distribution throughout Japan, including 7 hospitals in Eastern Japan and 10 hospitals in Western Japan. These 17 hospitals included 13 university hospitals and 4 community hospitals. Data were collected by these participating hospitals from patients $\geq 18$ years who were diagnosed with 'classical fever of unknown origin' between 1 January and 31 December 2011. The data were recorded on standardised case report forms and were collected by fax.

Classical FUO was diagnosed based on the definition by Durack et a ${ }^{\ominus}$ in patients meeting all of criteria $1-4$ below.

1. Fever with axillary temperature $\geq 38^{\circ} \mathrm{C}$ at least twice over a $\geq 3$-week period.

2. Unknown cause after three outpatient visits or during 3 days of hospitalisation.

3. Not diagnosed with immunodeficiency before fever onset.

4. No confirmed HIV infection before fever onset.

Axillary temperature is usually measured in Japan, so fever was defined as a temperature $\geq 38^{\circ} \mathrm{C}$.

The data described below were collected. No additional testing was performed in this study due to insufficient data.

- Patient characteristics: sex, age, concomitant disease, medical history and medication history.

- Clinical findings: subjective symptoms and objective physical findings.

- Blood tests: blood count, biochemical examination and inflammatory markers $(\mathrm{C}$ reactive protein (CRP), erythrocyte sedimentation rate (ESR), procalcitonin).

- Results of blood cultures if performed.

- Results of imaging studies and endoscopy if performed.

- Results of cytology, histology, genetic testing or autopsy findings if performed.

- Final diagnosis, day of diagnosis and outcome.

\section{RESULTS}

A total of 121 patients with FUO were enrolled at the 17 participating hospitals. There were 52 women (43.0\%), and the median patient age was 59 years (range 1994 years). The most frequent age group was patients in their 70s (figure 1). There were 112 patients from 13 university hospitals and 9 patients from 4 community hospitals.

Causative diseases for FUO were infectious disease in $28(23.1 \%)$ patients, non-infectious inflammatory

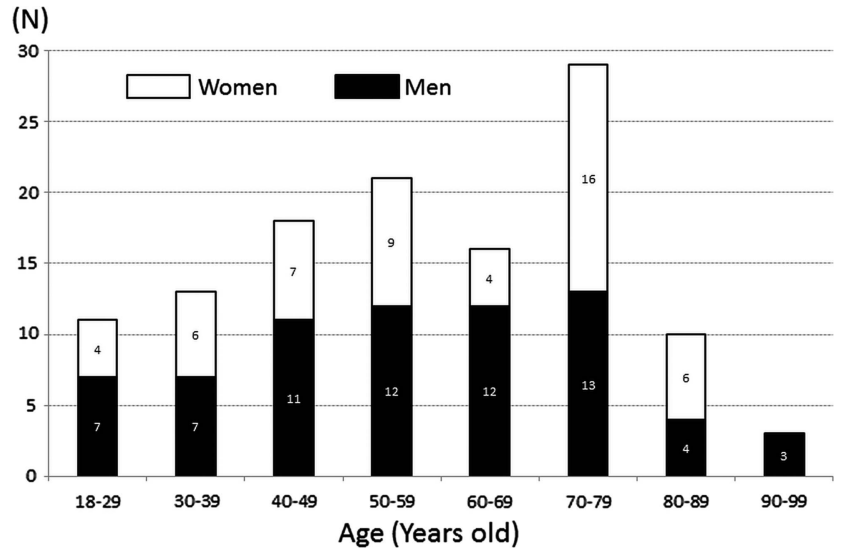

Figure 1 Age and sex distribution of fever of unknown origin patients.

disease (NIID) in $37(30.6 \%)$, malignancy in 13 $(10.7 \%)$, other in $15(12.4 \%)$ and unknown in 28 (23.1\%; figure 2). The most common causative disease was polymyalgia rheumatica (PMR; 9 patients), followed by malignant lymphoma ( 8 patients) and adult Still's disease ( 7 patients). Among the nine patients with PMR, coexisting giant cell arteritis was ruled out by PET in four patients and temporal artery biopsy in one patient. In the remaining four patients, symptoms and the success of treatment with low-dose steroids suggested PMR alone. Table 1 lists the frequent causative diseases. The prognosis in patients with undiagnosed FUO has been reported as relatively good. ${ }^{10}$ In our study as well, among 28 patients with undiagnosed FUO, only 3 died $(10.7 \%)$. Among the three patients who died with undiagnosed FUO, one was a 72-year-old man who developed disseminated intravascular coagulation (DIC) 2 months after fever onset and died. Another was an 82-year-old man who similarly developed DIC of unknown cause 1 month after fever onset and died. The other was a 63-year-old woman in whom the cause of FUO remained unknown despite PET and random skin biopsies. This patient developed respiratory failure about 6 months after fever onset and died.

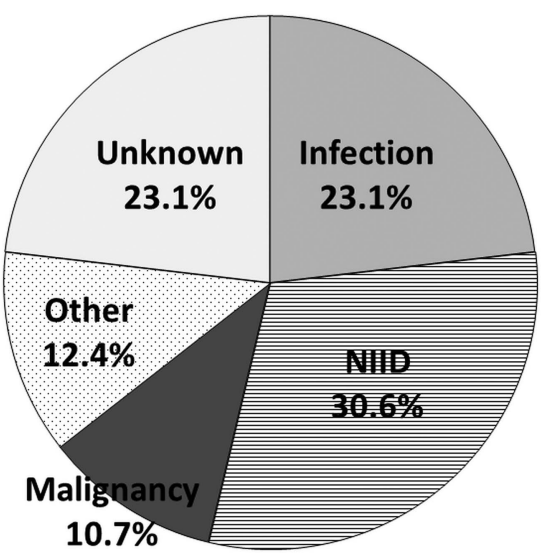

Figure 2 Causative disease in fever of unknown origin. NIID, non-infectious inflammatory disease. 


\begin{tabular}{lc} 
Table 1 Frequent causes & \\
\hline Causes & Number (\%) \\
\hline Infection & $28(23.1)$ \\
Infectious endocarditis & 4 \\
HIV/AIDS & 4 \\
Tuberculosis & 3 \\
Other & 17 \\
NIID & $37(30.6)$ \\
Polymyalgia rheumatica & 9 \\
Still's disease & 7 \\
Sarcoidosis & 3 \\
ANCA-associated vasculitis & 3 \\
Rheumatoid arthritis & 3 \\
Other & 12 \\
Malignancy & $13(10.7)$ \\
Malignant lymphoma & 8 \\
Castleman's disease & 2 \\
Other & 3 \\
Other & $15(12.4)$ \\
Drug fever & 3 \\
Fraudulent fever & 3 \\
Other & 9 \\
Unknown & $28(23.1)$ \\
\hline ANCA, anti-neutrophil cytoplasmic antibody; NIID, non-infectious \\
inflammatory disease;. \\
\end{tabular}

In patients $\geq 65$ years ( 51 patients), malignancy as the causative disease was twice as common as in patients $<65$ years, and the cause remained unknown in only a few of these patients (figure 3).

The median duration from fever onset to evaluation at each hospital was 28 days. Among the 24 patients requiring $\geq 100$ days from fever onset until diagnosis, 10 patients $(41.7 \%)$ had malignancy, exceeding the number with infection (7 patients, $29.2 \%$; figure 4 ). Among patients with malignancy, $60 \%$ had malignant lymphoma. The longest time required for diagnosis was a case of familial Mediterranean fever, which required $\geq 2$ years from the time of fever onset for diagnosis.

Tests performed for diagnostic evaluation included blood cultures in $86.8 \%$, serum procalcitonin in $43.8 \%$, gallium scintigraphy in $31.4 \%$ and PET in $29.8 \%$ of patients (figure 5). However, only 1 of 17 patients $(5.9 \%)$ with a serum procalcitonin level $\geq 0.25 \mathrm{ng} / \mathrm{mL}$ showed bacterial infection and 3 patients $(11.5 \%)$ with a value $<0.25 \mathrm{ng} / \mathrm{mL}$ also had bacterial infection.

Nine deaths occurred among all 121 patients. The most common cause was malignant lymphoma, in four patients. Pathological autopsy was performed on a small proportion of the patients who died $(n=2 ; 22.2 \%)$. The diagnosis was malignant lymphoma in both of these autopsied cases.

\section{DISCUSSION}

Causative diseases underlying FUO vary by region and time period, and evaluation of these causes over a wide area using recent data is important. A previous study in Japan reported tuberculosis as the most common cause
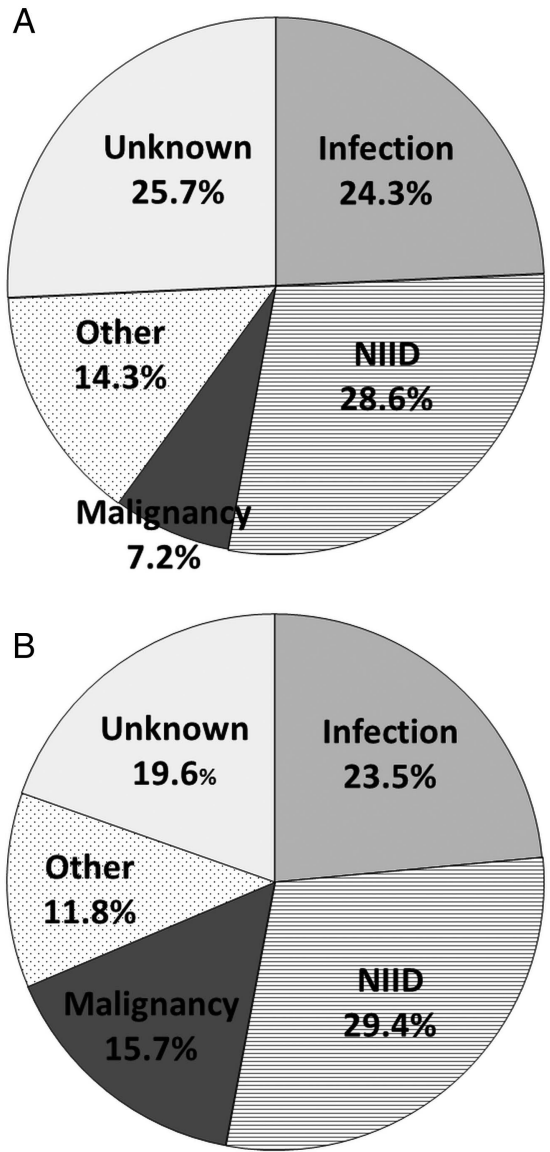

Figure 3 (A) Causative disease in patients $<65$ years (70 patients). (B) Causative disease in patients $\geq 65$ years (51 patients).

of FUO, accounting for $10 \%$ of cases. $^{7}$ The present study identified NIID as the most common cause. Infections, including tuberculosis, were diagnosed based on appropriate culture or serological tests, before meeting the definition of classical FUO.

On the basis of the results of a survey conducted by the Organisation for Economic Cooperation and Development (OECD) in 30 member countries, Japan has 92.6 CT scanners and 40.1 MRI scanners per million population, the highest among OECD member countries. ${ }^{11}$ Because CT and MRI tend to be performed early after fever onset, FUO due to deep-seated abscess and solid tumours has decreased markedly. Among our 121 patients, the only deep-seated abscess was an ovarian abscess found in one patient, and the solid tumours were renal carcinoma in one patient and anal canal carcinoma in one patient only.

PMR was the most common disease causing FUO (9 patients) in the present study. This was probably influenced by the ageing of the Japanese population. According to data from the Ministry of Internal Affairs and Communications Statistics Bureau, among the total population in Japan of 127.34 million, 31.4 million $(24.7 \%)$ are elderly persons $\geq 65$ years, the highest number ever. ${ }^{12}$ In our study, $42.1 \%$ of patients were 


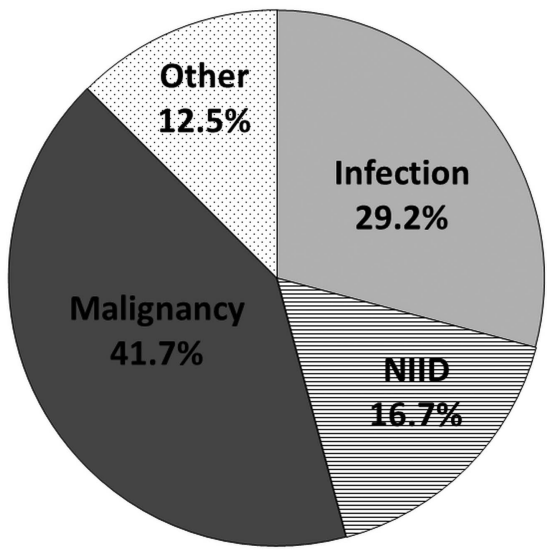

Figure 4 Causative disease in patients requiring $\geq 100$ days until diagnosis (24 patients).

$\geq 65$ years. A diagnosis of PMR, which occurs with a relatively high incidence in elderly patients, must thus be considered for FUO. Moreover, this trend should also be considered in Western countries, where ageing of the population is also expected. PMR had an influence, and when participants $\geq 65$ and $<65$ years were compared, rates of NIID as a cause of FUO were similar (figure 3).

In Japan, 1529 new HIV infections were reported during 2011, ${ }^{13}$ and this increase in HIV-infected persons is continuing. Our study also found four patients with FUO caused by HIV/AIDS. The importance of HIV/ AIDS as a cause of FUO will probably continue to increase in Japan. In our study, patients already diagnosed with HIV infections before meeting the definition of classical FUO were excluded.

Evaluations vary regarding the usefulness of ESR to diagnose FUO. ${ }^{6}$ Well-known diseases associated with ESR $>100 \mathrm{~mm} / \mathrm{h}$ include PMR, tuberculosis, multiple myeloma and osteomyelitis (vertebral discitis), but malignancies have also been reported in about $60 \%$ of such cases. ${ }^{14}$ In our study, 18 of 121 patients (14.9\%) had an

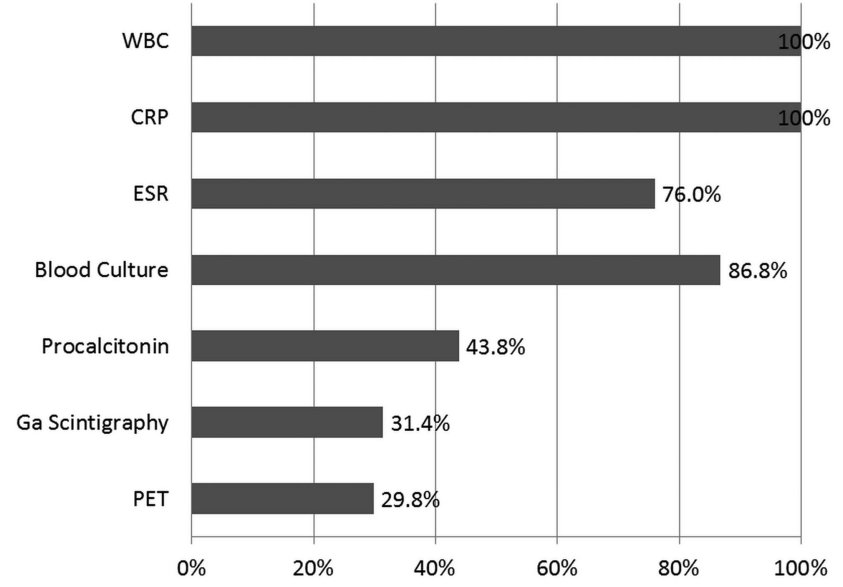

Figure 5 Frequency of tests performed for diagnostic evaluation. WCC, white blood cells count; CRP, $C$ reactive protein; ESR, erythrocyte sedimentation rate; Ga, gallium; PET, positron emission tomography.
ESR of $100 \mathrm{~mm} / \mathrm{h}$, including 5 with unknown cause, 2 with PMR and 2 with ANCA-associated vasculitis.

When evaluating FUO, performing differential diagnosis for bacterial infections is important, given the need for prompt antibiotic therapy. It goes without saying that blood cultures are essential. In patients with bacteraemia given inappropriate treatment without performing blood cultures, the mortality rate has been reported as 3.18 times higher compared with patients with initial appropriate treatment. However, if treatment is switched based on blood culture results, the mortality rate is only 1.27 times higher. ${ }^{15}$

In recent years, the usefulness of serum procalcitonin in the differential diagnosis of bacterial infections has increasingly been reported, and serum procalcitonin is now in wide use in Japan. Simon et al reported that for differential diagnosis of bacterial infection from non-infectious disease, serum procalcitonin offers higher sensitivity $(88 \%)$ and specificity $(81 \%)$ than CRP (75\% and $67 \%$, respectively). ${ }^{16} \mathrm{In}$ our study, serum procalcitonin was measured in $43.8 \%$ of patients. Although use of this test is widespread in Japan, $5.9 \%$ of patients with a serum procalcitonin level $\geq 0.25 \mathrm{ng} /$ $\mathrm{mL}$ showed bacterial infection, and $11.5 \%$ of patients with a value $<0.25 \mathrm{ng} / \mathrm{mL}$ also had bacterial infection. It is, therefore, difficult to conclude that serum procalcitonin is a superior test for the differential diagnosis of bacterial infection in patients with classical FUO. Serum procalcitonin testing is also more expensive than CRP or ESR testing, so indiscriminate measurement of serum procalcitonin in febrile patients should be avoided.

PET appears promising as a useful test to evaluate FUO. Many studies have been performed using this modality in patients with FUO, all showing a percentage helpfulness that exceeds that of CT, MRI or other diagnostic possibilities. ${ }^{17-20}$ However, the fact that PET is not covered by National Health Insurance in Japan for patients with FUO is problematic. A previous Japanese study about PET included patients with FUO evaluated by radiology departments. ${ }^{21}$ In the present study, it was interesting to see the frequency of PET use in general medical settings, mainly for evaluating FUO.

Previous studies have reported increasing rates of FUO in which the cause remains unknown. ${ }^{22} 23$ A prospective study from the Netherlands reported that the cause of FUO remained unknown in $51 \%$ of cases. ${ }^{6}$ The rate of 'unknown' in our study was relatively small, but this retrospective study may have encountered bias because of the difficulty enrolling patients with an unknown cause after undergoing tests. Furthermore, retrospective studies also encounter the problem that there may be no follow-up observation of patients until a final diagnosis has been obtained. We are planning a prospective study of patients who are enrolled at the time when criteria for FUO are fulfilled, to perform periodic follow-up observations. Only 17 hospitals participated in this study, so the results may not be generalisable to the overall situation in Japan. We hope that more hospitals will participate in future studies. 


\section{CONCLUSION}

We conducted the first nationwide study of patients with FUO in Japan. Our study identified diseases that should now be considered in the differential diagnosis for FUO. This will be useful in future clinical practice. In addition, we identified tests that are performed during the FUO evaluation process at each hospital. This will serve as a reference for selecting diagnostic tests in the future. Guidelines should be created based on this type of study for future evaluation of FUO.

\section{Author affiliations}

${ }^{1}$ Department of General Medicine, Juntendo University School of Medicine, Tokyo, Japan

${ }^{2}$ Department of General Internal Medicine, Hiroshima University Hospital, Hiroshima, Japan

${ }^{3}$ Department of General Internal Medicine, Kyushu University Hospital, Fukuoka, Japan

${ }^{4}$ Department of General Internal Medicine, Kobe University Hospital, Kobe, Japan

${ }^{5}$ Department of General Internal Medicine, St Marianna University School of Medicine, Kawasaki, Japan

${ }^{6}$ Department of General Medicine, Nara Medical University, Nara, Japan ${ }^{7}$ Department of General Internal Medicine, Gifu University Graduate School of Medicine, Gifu, Japan

${ }^{8}$ Department of General Medicine, Osaka Medical College, Osaka, Japan ${ }^{9}$ Department of General Internal Medicine, Kainan Hospital, Aichi Prefectural Welfare Federation of Agricultural Cooperatives, Aichi, Japan

${ }^{10}$ Department of General Medicine, Okayama University Graduate School of

Medicine, Dentistry and Pharmaceutical Sciences, Okayama, Japan

${ }^{11}$ Department of General Medicine, Gunma University Graduate School of Medicine, Gunma, Japan

${ }^{12}$ Department of General Medicine, Yokohama City University Medical Center, Yokohama, Japan

${ }^{13}$ Department of General Medicine, Faculty of Medicine, Oita University, Oita, Japan

${ }^{14}$ Department of General Medicine, Kitakyushu Municipal Medical Center, Kitakyushu, Japan

${ }^{15}$ Department of Internal Medicine, Shonan Fujisawa Tokushukai Hospital, Fujisawa, Japan

${ }^{16}$ Department of General Medicine, Sumitomo Hospital, Osaka, Japan

${ }^{17}$ Department of Integrated Medicine, Kagawa University School of Medicine, Kagawa, Japan

Contributors TN, MM, AU, NM, FO, SS, ST and JH conceived and designed the study. TN, MM, FM, KK, KT, SO, HM, AU, NM, FO, YO, NN, KM, KM, KA, $\mathrm{KY}$ and SS were involved in acquisition of data. TN, MM, FM, KK, KT, SO, $\mathrm{HM}, \mathrm{AU}, \mathrm{NM}, \mathrm{FO}, \mathrm{YO}, \mathrm{NN}, \mathrm{KM}, \mathrm{KM}, \mathrm{KA}, \mathrm{KY}$ and SS analysed the data. TN, KT and MY wrote the manuscript.

Funding Grant-in-Aid from MEXT (Ministry of Education, Culture, Sports, Science and Technology) Supported Program for the Strategic Research Foundation at Private Universities, 2012-2017.

Competing interests None.

Ethics approval Research Ethics Committee of Juntendo University School of Medicine.

Provenance and peer review Not commissioned; externally peer reviewed.

Data sharing statement No additional data are available.

Open Access This is an Open Access article distributed in accordance with the Creative Commons Attribution Non Commercial (CC BY-NC 3.0) license, which permits others to distribute, remix, adapt, build upon this work non-commercially, and license their derivative works on different terms, provided the original work is properly cited and the use is non-commercial. See: http://creativecommons.org/licenses/by-nc/3.0/

\section{REFERENCES}

1. Alt $\mathrm{HL}$, Barker $\mathrm{MH}$. Fever of unknown origin. JAMA 1930;94:1457-61.

2. Petersdorf RG, Beeson PB. Fever of unexplained origin: report on 100 cases. Medicine (Baltimore) 1961;40:1-30.

3. de Kleijn EM, Vandenbroucke JP, van der Meer JW. Fever of unknown origin (FUO). I A. prospective multicenter study of 167 patients with FUO, using fixed epidemiologic entry criteria. The Netherlands FUO Study Group. Medicine (Baltimore) 1997;76:392-400.

4. Miller RF, Hingorami AD, Foley NM. Pyrexia of undetermined origin in patients with human immunodeficiency virus infection and AIDS. Int J STD AIDS 1996;7:170-5.

5. Zenone T. Fever of unknown origin in adults: evaluation of 144 cases in a non-university hospital. Scand J Infect Dis 2006;38:632-8.

6. Bleeker-Rovers CP, Vos FJ, de Kleijn EM, et al. A prospective multicenter study on fever of unknown origin: the yield of a structured diagnostic protocol. Medicine (Baltimore) 2007;86:26-38.

7. Shoji S, Imamura A, Imai Y, et al. Fever of unknown origin: a review of 80 patients from the Shin'etsu area of Japan from 1986-1992. Intern Med 1994;33:74-6.

8. likuni $\mathrm{Y}$, Okada $\mathrm{J}$, Kondo $\mathrm{H}$, et al. Current fever of unknown origin 1982-1992. Intern Med 1994;33:67-73.

9. Durack DT, Street AC. Fever of unknown origin: re-examined and redefined. Current clinical topics in infectious diseases. Remington, JS: Blackwell Science, 1991:35-51.

10. Knockaert DC, Dujardin KS, Bobbaers HJ. Long-term follow-up of patients with undiagnosed fever of unknown origin. Arch Intern Med 1996;156:618-20.

11. Health at a Glance 2009: OECD Indicators. http://www.oecd-ilibrary. org/sites/health_glance-2009-en/04/03/index.html? contentType $=\&$ itemld $=/$ content/chapter/health glance-2009-38-en\&containerltemld=/content/serial/ 19991312\&accessltemlds=/content/book/health glance-2009-en\&mimeType=text/html (accessed 12 Oct 2013).

12. Statistics Bureau, Director-General for Policy Planning, Statistical Research and Training Institute. http://www.stat.go.jp/english/data/ jinsui/tsuki/index.htm (accessed 12 Oct 2013).

13. AIDS list of statistical surveys conducted by the Ministry of Health, Labour and Welfare. 2011. http://api-net.jfap.or.jp/status/2011/ 11nenpo/hyo 01.pdf (accessed 12 Oct 2013).

14. Zacharski LR, Kyle RA. Significance of extreme elevation of erythrocyte sedimentation rate. JAMA 1967;202:264-6.

15. Weinstein MP, Towns ML, Quartey SM, et al. The clinical significance of positive blood cultures in the 1990s: a prospective comprehensive evaluation of the microbiology, epidemiology, and outcome of bacteremia and fungemia in adults. Clin Infect Dis 1997;24:584-602.

16. Simon L, Gauvin F, Amre DK, et al. Serum procalcitonin and C-reactive protein levels as markers of bacterial infection: a systematic review and meta-analysis. Clin Infect Dis 2004;39:206-17.

17. Bleeker-Rovers CP, Vos FJ, Mudde AH, et al. A prospective multi-centre study of the value of FDG-PET as part of a structured diagnostic protocol in patients with fever of unknown origin. Eur J Nucl Med Mol Imaging 2007;34:694-703.

18. Meller J, Altenvoerde G, Munzel U, et al. Fever of unknown origin: prospective comparison of [18F] FDG imaging with a double-head coincidence camera and gallium-67 citrate SPET. Eur J Nucl Med 2000;27:1617-25.

19. Kouijzer IJ, Bleeker-Rovers CP, Oyen WJ. FDG-PET in fever of unknown origin. Semin Nucl Med 2013;43:333-9.

20. Meller J, Sahlmann CO, Scheel AK. 18F-FDG PET and PET/CT in fever of unknown origin. J Nucl Med 2007;48:35-45

21. Kubota K, Nakamoto Y, Tamaki N, et al. FDG-PET for the diagnosis of fever of unknown origin: a Japanese multi-center study. Ann Nucl Med 2011;25:355-64.

22. Horowitz HW. Fever of unknown origin or fever of too many origins? N Engl J Med 2013;368:197-9.

23. Mourad O, Palda V, Detsky AS. A comprehensive evidence-based approach to fever of unknown origin. Arch Intern Med 2003;163:545-51. 